\title{
Demanda social y perfil del Ingeniero Ambiental en la Universidad Nacional «Santiago Antúnez de Mayolo»
}

\author{
Social demand and profile Environmental Engineering at the «Santiago \\ Antúnez de Mayolo» National University \\ Paula Falcón Romero ${ }^{1}$
}

\section{RESUMEN}

El objetivo es determinar la relación que existe entre la demanda social y el perfil profesional del egresado de la carrera de Ingeniería Ambiental de la Facultad de Ciencias del Ambiente de la Universidad «Santiago Antúnez de Mayolo». El tipo de investigación fue descriptivo correlacional, la población estuvo constituida por 15 empresas de la Región Áncash, zona sierra y 99 egresados hábiles del Colegio de Ingenieros, filial Huaraz. Los resultados de la investigación con respecto a la correlación entre la demanda de trabajo y las áreas del perfil profesional, recursos naturales y biodiversidad; gestión ambiental; prevención, control y remediación ambiental; educación ambiental indicaron que los coeficientes de correlación de Pearson hallados 0.778, 0.754, 0.699, 0.687 y han demostrado que existe relación positiva y media-alta. Son significativas al 5\%, la significación bilateral resultó 0.000 menor de 0.05 en todos los casos. Existe relación directa, lo que demostró que los contenidos temáticos y prácticos de las áreas satisfacen la demanda de trabajo pero que es necesario reforzarlos para un mejor desempeño. El perfil profesional que se sugiere presentó algunas modificaciones y nuevas áreas para el perfil profesional.

Palabras clave: demanda social; demanda de trabajo; desempeño profesional; perfil profesional.

\begin{abstract}
The research has had as general objective to determine the relationship between social demand and professional profile of graduates of Environmental Engineering, Faculty of Environmental Sciences of the University «Santiago Antunez de Mayolo». The research was descriptive correlational, the population consisted of 15 companies -zona Ancash region saw 99 business graduates Engineers Association, subsidiary- Huaraz. The results of the research regarding the correlation between demand for labor and professional profile areas of natural resources and biodiversity; environmental
\end{abstract}

1 Universidad Nacional «Santiago Antúnez de Mayolo». Huaraz, Perú. 
management; prevention, control and environmental remediation; environmental education indicated that Pearson correlation coefficients found 0.778, 0.754, 0.699, 0.687 showed that there is positive and medium-high relationship. They are significant at the $5 \%$ significance it was bilateral 0.000 less than 0.05 in all cases. Direct relationship exists demonstrating that the thematic and practical content areas satisfy the demand for labor but need to be strengthened for better performance. The professional profile suggested introduced some modifications and new areas for professional profile.

Keywords: social demand; demand for labor; professional performance; professional profile.

\section{ICHIKLLACHAW}

Kay yachay ashiy munarqan universidad Santiago Antúnez de Mayolochaw, Escuela de Ingeniería Ambiental de la Facultad de Ciencias del Ambientepita yachakuyninta yarquqkunapa markantsikchaw paykunapita shuyashqantsik imanawpis kashqanta. Kay yachay ashiy kashqa descriptivo correlacionalmi, tsaypaq tsariyarquu Ancash regionpa-zona sierranchawmi 15 empresakunata, 99 yachaynin ushashqata, Colegio de Ingenierusman yayakuyaananpaq mañakuykaqta. Kaychawmi tarikashqa demanda de trabajuwan áreas del perfil profesional recursos naturaleswan biodiversidad; gestión ambiental; prevención, control y remediación ambiental; educación ambiental willakushqa coeficientes de correlación de Pearson hallados 0.778, 0.754, 0.699, 0.687 willakushqa relación positivawan media-altata. Alli kaqmi 5\%, la significación bilateral yarqamushqa 0.000 menor de 0.05 llapan uryashqachaw. Ishkan tumaqkunam tanqanakuyan.

Pushaq shimikuna: marka mayintsikkunapa mañakuynin; uryapaq kaq mañakuy; uryashqantsikchaw imapis alli ruray; profesional kashqantsik willakuq.

\section{INTRODUCCIÓN}

El currículo de estudios de la Facultad de Ciencias Ambientales de la Universidad Nacional «Santiago Antúnez de Mayolo» fue reestructurado por la Comisión de Reestructuración Curricular en el año 2006. En su aplicación, hubo una serie de dificultades: no estaba implementado con un adecuado porcentaje de competencias genéricas, instrumentales sistémicas, interpersonales, tecnológicas generales, no respondía eficientemente a las demandas de los empleadores, por ello la comisión curricular recomendó que el currículo debía ser mejorado.

El problema de la investigación fue ¿qué relación existe entre la demanda social actual y el perfil profesional de los egresados de la carrera profesional de Ingeniería Ambiental de la Universidad Nacional «Santiago Antúnez de Mayolo»?

La justificación radica en que los currículos actuales de la UNASAM no se han realizado sobre la base de competencias, sino a los objetivos profesionales, sin un estudio de las demandas sociales identificadas por la demanda de trabajo por áreas del perfil profesional y desempeño profesional de los egresados.

El objetivo fue determinar el nivel de correlación que existe entre la demanda social y 
el perfil profesional del egresado. Los objetivos específicos fueron determinar la relación entre la demanda de trabajo y las cuatro áreas del perfil profesional, sugerir un perfil profesional reestructurado de la carrera de Ingeniería Ambiental.

Al respecto del perfil profesional Fundación Fórum Ambiental (2001) concluyó que el perfil ideal del ingeniero ambiental tendría las siguientes características:

Titulación universitaria con estudios de especialización, con experiencia a nivel directivo y tendría las competencias técnicas, estratégicas, organizativas, transversales y relacionales.

Sota et al. (2000: 12) comentaron que:

La universidad necesita cambiar sus perfiles académicos y profesionales para hacerlos más integrales. El perfil profesional es la descripción de las ocupaciones existentes en el sector del empleador y que están siendo o se espera sea desempeñado por el egresado de un programa o trabajador, tratando de establecer la relación cargo-función-responsabilidad como también los componentes actitudinales, habilidades y destrezas que se requiere para el desempeño de dicho cargo.

«La creación de un perfil ocupacional se puede considerar una parte del análisis y la descripción de cargos, ya que a partir de las necesidades empresariales, se crean perfiles ocupacionales como un elemento en la selección y análisis de personal» (Sánchez, 2003: 8). Otro concepto de demanda social dado por Hawes y Corvalán (2005: 83) indica que más próximo a una determinación basada en las necesidades educativas de las personas está el concepto de demanda social definido como «la demanda de educación que surge de los deseos y aspiraciones de los individuos».

Las nuevas demandas de los mercados laborales, cada vez más diferenciados y versátiles, obligan a las instituciones de educación superior a ser funcionales a sus necesidades, instruyendo profesionales competentes en oficios, ocupaciones y profesiones de modo tal que se facilite su inserción en el mercado laboral (Brunner, 2001).

La Guía de Reestructuración Curricular UNASAM (2010) indica que el estudio de la demanda social constituye una investigación en el campo laboral, por el cual se recogerá información de los requerimientos y áreas de desempeño del medio laboral en el cual se desenvuelven los egresados. Para los efectos se consulta con los egresados, profesionales reconocidos del área, empleadores o cualquier otro actor que facilite información.

\section{MATERIALES Y MÉTODOS}

El tipo de investigación es descriptivo - correlacional; el diseño, no experimental, transecional, año 2012. El plan de recolección de la información y/o diseño estadístico tuvo como población 15 empresas donde se desempeñan profesionalmente ingenieros ambientales o con grado de maestro y/o doctor. La muestra de empresas fue no probabilística, intencional. También se consideró a los egresados de la carrera de Ingeniería Ambiental de la UNASAM hábiles en el Colegio de Ingenieros del Perú Filial-Huaraz (en total 99 incluidos los profesionales que se desempeñan como docentes de la carrera en la Facultad de Ciencias del Ambiente). La muestra de egresados estuvo 
constituida por 79 egresados. La técnica de la revisión bibliográfica, se ha llevado a cabo según la temática que corresponde al marco conceptual y teórico, y el currículo de estudios vigente de la Facultad de Ingeniería Ambiental de la UNASAM. La técnica de la entrevista se ha aplicado mediante los cuestionarios para obtener información de los egresados y empleadores, para la tabulación de la información utilizando un software SPSS versión 21. Los instrumentos de recolección de la información han sido las encuestas, fueron analizadas mediante la prueba estadística de hipótesis paramétrica del Coeficiente de Correlación de Pearson.

\section{RESULTADOS}

Los resultados del estudio de la demanda social, el campo laboral, trayectoria profesional de la muestra de egresados de la carrera de ingeniería ambiental por áreas del perfil profesional del currículo vigente, se muestran en la tabla 1 y los resultados del estudio de la relación entre la demanda de trabajo y las áreas del perfil profesional se presentan en las tablas 2, 3, 4, 5 donde aparecen las medidas de la correlación entre las variables estudiadas. En la tabla 6 se indica el perfil reestructurado.

Tabla 1. Ocupación laboral de la muestra de egresados por área del perfil profesional, instituciones y sector al que pertenecen en el año 2012

\begin{tabular}{|c|c|c|c|c|c|}
\hline Institución & $\begin{array}{l}\text { Número de } \\
\text { egresados - } \\
\text { colegiados } \\
\end{array}$ & Sector & Área de desempeño & $\mathrm{N}^{\circ}$ & $\%$ \\
\hline Universidad & $\begin{array}{l}13 \text { En Huaraz } \\
4 \text { En otras Universi- } \\
\text { dades del Perú }\end{array}$ & $\begin{array}{l}\text { Público y Pri- } \\
\text { vado }\end{array}$ & $\begin{array}{l}\text { Enseñanza } \\
\text { Gestión ambiental }\end{array}$ & 17 & 21,5 \\
\hline $\begin{array}{l}\text { Instituciones del } \\
\text { gobierno, regional } \\
\text { municipal distrital }\end{array}$ & 30 & Público & $\begin{array}{l}\text { Gestión ambiental, } \\
\text { Recursos naturales } \\
\text { y Biodiversidad }\end{array}$ & $\begin{array}{l}3 \\
1\end{array}$ & 39,2 \\
\hline Consultoría privada & 18 & Privado & Gestión ambiental & 18 & 22,8 \\
\hline Empresas mineras & 10 & Privado & $\begin{array}{l}\text { Prevención y con- } \\
\text { trol y remediación } \\
\text { ambiental } \\
\text { Gestión ambiental }\end{array}$ & 10 & 12,7 \\
\hline $\begin{array}{l}\text { Otras Empresas } \\
\text { comerciales hidráu- } \\
\text { licas }\end{array}$ & 3 & $\begin{array}{l}\text { Privado, contra- } \\
\text { to temporal }\end{array}$ & Gestión ambiental & 3 & 3.8 \\
\hline Total & 79 & ----- & ----- & 79 & 100,0 \\
\hline
\end{tabular}

Lo indicado en la tabla 1 demuestra que los egresados se desempeñan en las cuatro áreas del perfil. Las instituciones públicas absorben el mayor número de profesionales, $39.2 \%$, le siguen las ocupaciones de consultoría privada en el $22 \%$; el rubro de enseñanza en un $21.5 \%$. 
Tabla 2. Medidas de correlación entre la Demanda de trabajo y el área de Recursos Naturales y Biodiversidad

\begin{tabular}{llcc}
\hline \multicolumn{1}{c}{ Variables } & \multicolumn{1}{c}{ Estadígrafo } & $\begin{array}{c}\text { Demanda de } \\
\text { trabajo }\end{array}$ & $\begin{array}{c}\text { Área de Recursos } \\
\text { naturales y } \\
\text { biodiversidad }\end{array}$ \\
\hline Demanda de trabajo & $\begin{array}{l}\text { Correlación Pearson } \\
\text { Sig. (Bilateral }\end{array}$ & 1.000 & 0.778 \\
& $\mathrm{~N}$ & & 0000 \\
& & 79 & 79 \\
$\begin{array}{l}\text { Área de recursos } \\
\text { naturales y } \\
\text { biodiversidad }\end{array}$ & $\begin{array}{l}\text { Correlación Pearson } \\
\text { Sig. (Bilateral }\end{array}$ & 0.778 & 1.000 \\
\hline
\end{tabular}

Tabla 3. Medidas de correlación entre la Demanda de trabajo y el área de Gestión Ambiental de los egresados de la carrera de Ingeniería Ambiental.

\begin{tabular}{llcc}
\hline \multicolumn{1}{c}{ Variables } & \multicolumn{1}{c}{ Estadístico } & $\begin{array}{c}\text { Demanda de } \\
\text { trabajo }\end{array}$ & $\begin{array}{c}\text { Área de gestión } \\
\text { ambiental }\end{array}$ \\
\hline Demanda de trabajo & $\begin{array}{l}\text { Correlación Pearson } \\
\text { Sig. (Bilateral }\end{array}$ & 1.000 & 0.754 \\
& $\mathrm{~N}$ & 79 & 0000 \\
& Correlación Pearson & 0.754 & 79 \\
$\begin{array}{l}\text { Área de gestión } \\
\text { ambiental }\end{array}$ & Sig. (Bilateral & 0000 & 1.000 \\
& $\mathrm{~N}$ & 79 & 79 \\
\hline
\end{tabular}

Tabla 4. Medidas de correlación entre la demanda de trabajo y el área del perfil profesional: Prevención y Control de la Remediación Ambiental

\begin{tabular}{llcc}
\hline \multicolumn{1}{c}{ Variables } & \multicolumn{1}{c}{ Estadístico } & $\begin{array}{c}\text { Demanda de } \\
\text { trabajo }\end{array}$ & $\begin{array}{c}\text { Área de prevención } \\
\text { y control de la } \\
\text { remediación } \\
\text { ambiental }\end{array}$ \\
\hline Demanda de trabajo & $\begin{array}{l}\text { Correlación Pearson } \\
\text { Sig. (Bilateral }\end{array}$ & 1.000 & 0.699 \\
& $\mathrm{~N}$ & 79 & 0000 \\
Área de prevención & Correlación Pearson & 0.699 & 79 \\
$\begin{array}{l}\text { y control de la } \\
\text { remediación ambiental }\end{array}$ & Sig. (Bilateral & 0000 & 1.000 \\
& $\mathrm{~N}$ & 79 & 79 \\
\hline
\end{tabular}


Tabla 5. Medida de correlación entre la demanda de trabajo y el área del perfil profesional Educación ambiental

\begin{tabular}{llcc}
\hline \multicolumn{1}{c}{ Variables } & \multicolumn{1}{c}{ Estadístico } & $\begin{array}{c}\text { Demanda de } \\
\text { trabajo }\end{array}$ & $\begin{array}{c}\text { Área de educación } \\
\text { ambiental }\end{array}$ \\
\hline Demanda de trabajo & $\begin{array}{l}\text { Correlación Pearson } \\
\text { Sig. (Bilateral }\end{array}$ & 1.000 & 0.687 \\
& $\mathrm{~N}$ & & 0000 \\
& & 79 & 79 \\
Área de educación & Correlación Pearson & 0.687 & 1.000 \\
ambiental & Sig. (Bilateral & 0000 & \\
& $\mathrm{~N}$ & 79 & 79 \\
\hline
\end{tabular}

En las tablas 2, 3, 4, 5, se aprecia los resultados de la investigación con respecto a la correlación entre la demanda de trabajo y las áreas del perfil profesional: recursos naturales y biodiversidad; gestión ambiental; prevención, control y remediación ambiental; educación ambiental.

Tabla 6. Perfil reestructurado del egresado de la carrera de Ingeniería Ambiental para ser implementado en una nueva versión del currículo

1. Introducción

En base al estudio de la demanda social en el año 2012, realizado en la región Áncash de la Zona Sierra, se determinó un perfil reestructurado de acuerdo a las funciones que desempeñan los ingenieros ambientales de la UNASAM en el año 2012.

Competencias generales

a) Aplica los conocimientos y principios del sistema de gestión integrado promoviendo el desarrollo sostenible del país en forma responsable.

b) Conoce y maneja eficientemente las NTIC's.

c) Gestiona la información para la generación de conocimientos.

d) Se integra a la comunidad y toma de decisiones en forma oportuna y apropiada para la solución de problemas a partir del conocimiento de la responsabilidad social y ética.

e) Utiliza la lengua materna y segunda lengua para la comunicación eficiente.

f) Desarrolla actitudes intra e interpretacionales sustentadas en el fortalecimiento de la inteligencia emocional, con una adecuada práctica de los valores morales.

g) Participa de forma asertiva, con liderazgo y responsabilidad en el trabajo de equipo

h) Promueve la convivencia en un contexto multicultural.

i) Genera conocimientos científicos vinculados a la realidad. 


\begin{tabular}{|c|c|c|c|}
\hline \multirow[b]{2}{*}{ Perfil } & \multicolumn{3}{|c|}{ Integración de desempeños y competencias } \\
\hline & Desempeños & $\begin{array}{l}\text { Competencias } \\
\text { específicas }\end{array}$ & $\begin{array}{l}\text { Capacidades y } \\
\text { actitudes }\end{array}$ \\
\hline \multirow{8}{*}{$\begin{array}{l}\text { Formación y } \\
\text { Educación Am- } \\
\text { biental }\end{array}$} & & $\begin{array}{l}\text { Orientar la enseñanza } \\
\text { por competencias y cen- } \\
\text { trada en el alumno } \\
\text { Aplicar nuevos modelos } \\
\text { organizativos y tecnolo- } \\
\text { gía educativa }\end{array}$ & $\begin{array}{l}\text { Autocontrol, tolerancia, ha- } \\
\text { bilidad para liderar y crear un } \\
\text { clima favorable para el apren- } \\
\text { dizaje del alumno } \\
\text { Responder a las necesidades } \\
\text { del alumno }\end{array}$ \\
\hline & Docencia & $\begin{array}{l}\text { Procedimientos de eva- } \\
\text { luación continua y for- } \\
\text { mativa de aprendizaje de } \\
\text { calidad }\end{array}$ & \multirow{2}{*}{$\begin{array}{l}\text { Monitoreo del proceso de } \\
\text { aprendizaje } \\
\text { Actitud de compromiso con el } \\
\text { aprendizaje, credibilidad, dis- } \\
\text { posición, empatía } \\
\text { compromiso y responsabilidad }\end{array}$} \\
\hline & & $\begin{array}{l}\text { Aplicar métodos para } \\
\text { estimar y regular la carga }\end{array}$ & \\
\hline & & $\begin{array}{l}\text { de trabajo para el alumno } \\
\text { y el profesor }\end{array}$ & \multirow{2}{*}{$\begin{array}{l}\text { Poseer valores } \\
\text { a) Inframorales } \\
\text { b) Instrumentales } \\
\text { c) Valores terminales }\end{array}$} \\
\hline & Instructor Facilitador & $\begin{array}{l}\text { Asesorar, diseñar progra- } \\
\text { mas de difusión, capaci- } \\
\text { tación y concientización }\end{array}$ & \\
\hline & Investigador & $\begin{array}{l}\text { Experiencia y conoci- } \\
\text { miento de las áreas bási- } \\
\text { cas y de especialidad de } \\
\text { la carrera }\end{array}$ & \multirow{3}{*}{$\begin{array}{l}\text { Habilidades sociales avanza- } \\
\text { das: } \\
\text { a) Orientadas a la comunica- } \\
\text { ción } \\
\text { b) Para trasmitir información, } \\
\text { persuadir, comprender y cum- } \\
\text { plir. Trabajo en equipo } \\
\text { c)Asertivas } \\
\text { d)Para afrontar situaciones } \\
\text { difíciles } \\
\text { e) Para negociar }\end{array}$} \\
\hline & $\begin{array}{l}\text { 1.Especialista en ma- } \\
\text { nejo de áreas natura- } \\
\text { les protegidas }\end{array}$ & $\begin{array}{l}\text { Conocimiento y manejo } \\
\text { sustentable de los recur- } \\
\text { sos naturales y foresta- }\end{array}$ & \\
\hline & $\begin{array}{l}\text { 2.Gestor ambiental } \\
\text { en recursos naturales } \\
\text { y de adaptación al } \\
\text { cambio climático }\end{array}$ & $\begin{array}{l}\text { Aplicación de sistemas de } \\
\text { información geográfica } \\
\text { y computarizada, gestión }\end{array}$ & \\
\hline \multirow[t]{3}{*}{$\begin{array}{l}\text { Recursos natu- } \\
\text { rales, biodiversi- } \\
\text { dad y gestión del } \\
\text { cambio climático }\end{array}$} & $\begin{array}{l}\text { 3. Gestor del riesgo } \\
\text { climático } \\
\text { 4.Supervisor de pro- } \\
\text { yectos ambientales }\end{array}$ & $\begin{array}{l}\text { de datos geográficos re- } \\
\text { ferenciados, utilizando } \\
\text { sistemas de hardware, } \\
\text { software (Geomedia pro- } \\
\text { fesional», «ARC-VIEW) }\end{array}$ & $\begin{array}{l}\text { Habilidad de Integración con } \\
\text { otros profesionales de la espe- } \\
\text { cialidad y afines a la temática } \\
\text { ambiental }\end{array}$ \\
\hline & $\begin{array}{l}\text { 5. Consultor y Eva- } \\
\text { luador de proyectos } \\
\text { ambientales }\end{array}$ & $\begin{array}{l}\text { y procedimientos dise- } \\
\text { ñados para soportar la } \\
\text { captura, gestión, manipu- } \\
\text { lación, análisis, modelado }\end{array}$ & $\begin{array}{l}\text { Disposición para aprender, } \\
\text { transferir y compartir conoci- } \\
\text { mientos tecnológicos. }\end{array}$ \\
\hline & $\begin{array}{l}\text { 6. Gerencia de re- } \\
\text { cursos naturales y } \\
\text { gestión del medio } \\
\text { ambiente }\end{array}$ & $\begin{array}{l}\text { y visualización de datos } \\
\text { espacialmente. }\end{array}$ & $\begin{array}{l}\text { Habilidades sociales: primarias } \\
\text { y avanzadas }\end{array}$ \\
\hline
\end{tabular}


Área de prevención y control de remediación ambiental

\begin{tabular}{|c|c|c|c|}
\hline \multirow[b]{2}{*}{ Perfil } & \multicolumn{3}{|c|}{ Integración de desempeños y competencias } \\
\hline & Desempeños & Competencias específicas & $\begin{array}{l}\text { Capacidades y } \\
\text { actitudes }\end{array}$ \\
\hline \multirow{3}{*}{$\begin{array}{l}\text { Prevención } \\
\text { control y } \\
\text { Remedia- } \\
\text { ción am- } \\
\text { biental }\end{array}$} & Gestor ambiental & $\begin{array}{l}\text { Dominio de metodologías y diag- } \\
\text { nóstico y evaluación del sanea- } \\
\text { miento y contaminación ambiental }\end{array}$ & \\
\hline & $\begin{array}{l}\text { Especialista en remediación } \\
\text { ambiental }\end{array}$ & $\begin{array}{l}\text { Dominio de metodologías de } \\
\text { monitoreo y vigilancia ambiental, } \\
\text { agua, suelo, aire } \\
\text { Control de remediación ambiental }\end{array}$ & \\
\hline & $\begin{array}{l}\text { Gestión de desastres natura- } \\
\text { les y/o industriales }\end{array}$ & $\begin{array}{l}\text { Diseña, instala, supervisa, opera } \\
\text { sistemas de prevención, tratamien- } \\
\text { to control y remediación ambiental }\end{array}$ & \\
\hline \multirow[t]{2}{*}{$\begin{array}{l}\text { Gestión } \\
\text { de riesgos, } \\
\text { seguridad y } \\
\text { salud ocu- } \\
\text { pacional }\end{array}$} & $\begin{array}{l}\text { Supervisor de seguridad y } \\
\text { medio ambiente }\end{array}$ & $\begin{array}{l}\text { Gestión de riesgos naturales y } \\
\text { procesos industriales, accidentes } \\
\text { de trabajo, salud ocupacional, ela- } \\
\text { boración de registros, accidentes } \\
\text { de trabajo, enfermedades ocupa- } \\
\text { cionales, incidentes peligrosos y } \\
\text { otros. También exámenes médicos } \\
\text { ocupacionales; monitoreo de agen- } \\
\text { tes físicos, químicos, biológicos, } \\
\text { psicosociales y factores de riesgo } \\
\text { disergonómicos; inspecciones in- } \\
\text { ternas de seguridad y salud en el } \\
\text { trabajo; estadísticas de seguridad } \\
\text { y salud; equipos de seguridad o } \\
\text { emergencia; inducción y simula- } \\
\text { cros de emergencia, y auditorías. }\end{array}$ & $\begin{array}{l}\text { Liderazgo, tra- } \\
\text { bajo en equipo, } \\
\text { adaptabilidad e } \\
\text { innovación comu- } \\
\text { nicacional }\end{array}$ \\
\hline & $\begin{array}{l}\text { Gestión de educación, salud } \\
\text { y medio ambiente en institu- } \\
\text { ciones públicas y privadas }\end{array}$ & & \\
\hline \multirow{3}{*}{$\begin{array}{l}\text { Sistemas de } \\
\text { Gestión }\end{array}$} & $\begin{array}{l}\text { Evaluador de impactos am- } \\
\text { bientales }\end{array}$ & $\begin{array}{l}\text { Conocimiento de la tecnología } \\
\text { ambiental industrial }\end{array}$ & \\
\hline & $\begin{array}{l}\text { Gestor de residuos, trata- } \\
\text { miento de aguas residuales, } \\
\text { contaminación atmosférica, } \\
\text { descontaminación de suelos, } \\
\text { diseño de instalaciones }\end{array}$ & & \\
\hline & Director de salud ambiental & $\begin{array}{l}\text { Responsable de áreas ecológicas, } \\
\text { saneamiento ambiental }\end{array}$ & \\
\hline
\end{tabular}




\begin{tabular}{|c|c|c|c|}
\hline \multirow{6}{*}{$\begin{array}{l}\text { Formación } \\
\text { básica }\end{array}$} & \multirow{6}{*}{$\begin{array}{l}\text { Capturar y asimilar conoci- } \\
\text { mientos formativos }\end{array}$} & \multicolumn{2}{|l|}{$\begin{array}{l}\text { Conocimientos básicos en ciencias } \\
\text { matemáticas, física, química }\end{array}$} \\
\hline & & $\begin{array}{l}\text { Conocimientos y aplicación de } \\
\text { técnicas de construcción, infraes- } \\
\text { tructura } \\
\text { Dinámica, estática, mecánica de } \\
\text { fluidos }\end{array}$ & $\begin{array}{l}\text { Actitud crítica, } \\
\text { capacidad de } \\
\text { análisis lógico } \\
\text { comprensión }\end{array}$ \\
\hline & & Geología & Habilidades so- \\
\hline & & $\begin{array}{l}\text { Conocimientos de estadística des- } \\
\text { criptiva }\end{array}$ & \\
\hline & & $\begin{array}{l}\text { Conocimientos y aplicación de } \\
\text { técnicas informática computari- } \\
\text { zadas }\end{array}$ & $\begin{array}{l}\text { Compromiso y } \\
\text { responsabilidad }\end{array}$ \\
\hline & & $\begin{array}{l}\text { Conocimientos básicos de admi- } \\
\text { nistración, contabilidad, economía }\end{array}$ & \\
\hline
\end{tabular}

En la tabla 6 se aprecia el perfil reestructurado con las competencias genéricas, capacidades y actitudes, competencias específicas por áreas del perfil reestructurado y por desempeño planteadas en la investigación como resultado de las respuestas de empleadores, egresados, docentes, etc.

\section{DISCUSIÓN}

Sobre la base de los resultados de las encuestas aplicadas a los empleadores y egresados relacionados con las funciones y cargos desempeñados, el campo laboral donde trabajan, el análisis del currículo actual, aspectos que constituyen el estudio de la demanda social, se elaboró el mapa funcional de la carrera, se identificó el propósito de cada área tal como recomienda la Guía de Reestructuración Curricular (2010). Se ha confirmado la hipótesis específica de investigación que dice que el estudio de la demanda orientará el diseño de un perfil reestructurado con competencias específicas para lograr un mayor desempeño. Sí hay demanda de Ingenieros Ambientales por parte de empresas locales. La trayectoria profesional ha sido utilizada también como un indicativo de la demanda de profesionales, el 89.9\% de los egresados encontró trabajo al término de sus estudios en la región Áncash y el 10.1 \% no (resultado de la inserción laboral de los egresados colegiados). El otro indicativo de la demanda es el porcentaje de egresados que encontraron trabajo en la región Áncash, en el período de las encuestas: el 81\% continuaba en la región Áncash (64 egresados colegiados) y el $17.7 \%$ de egresados trabajan fuera de la región Áncash. (14 egresados) y un egresado (1.3\%) se encontraba trabajando en el extranjero. Las áreas del perfil más demandadas fueron las de Gestión Ambiental y Prevención, Control y Remediación Ambiental, seguidas del área de Recursos Naturales y Biodiversidad. Esto concuerda con los estudios de Salas et al. (2004) que dicen que el área más demandada en el sistema europeo es la de sistemas 
de gestión ambiental en las organizaciones y el de consultoría y evaluación del impacto ambiental.

La relación entre la demanda de trabajo y las áreas del perfil Recursos Naturales y Biodiversidad y de Gestión Ambiental es positiva, los coeficientes de correlación fueron de 0.778 y de 0.754 valores cercanos que indican una correlación media alta, demostrándose que el contenido teórico -práctico de las áreas es aceptable para la demanda de trabajo, pero es necesario reforzar con capacidades específicas para mejorar la eficiencia en el desempeño profesional, implica también que existe un nivel adecuado en los egresados que les abre las puertas del mercado laboral.

Con respecto a la relación entre la demanda de trabajo y las áreas del perfil profesional existe relación entre la demanda social y el perfil profesional del currículo vigente. Los coeficientes de correlación de Pearson hallados 0.778, 0.754, 0.699, 0.687 demostraron que existe relación positiva y media-alta. Son significativos al 5\%. La significación bilateral resultó 0.000 menor de 0.05 en todos los casos. Esto se puede interpretar positivamente como que la preparación académica y práctica de las áreas satisface en un $77.8 \%, 75.4 \%, 69.9 \%, 68.7 \%$ a las demandas de trabajo de los empleadores; pero que es necesario mejorar para lograr un mejor desempeño. Se corrobora porque existe oferta de plazas de trabajo en el mercado local, nacional e internacional y ejercicio laboral aún antes de que los egresados se titulen. Con respecto al último objetivo de sugerir un perfil reestructurado para mejorar la eficiencia profesional con la introducción de competencias específicas, en Tobon (2006: 12) se ratifica la importancia de competencias específicas para la definición de cada área del perfil. Fuentes (2006: 50) expone que en las competencias profesionales están comprendidos los conocimientos, las habilidades y los valores profesionales.

\section{CONCLUSIONES}

Existe relación significativa entre la demanda social y el perfil del egresado de la carrera de ingeniería ambiental de la UNASAM, según la prueba de correlación de Pearson.

El análisis de la relación entre la Demanda de trabajo y el área de recursos naturales y biodiversidad alcanzan correlación estadística media -alta, directa y positiva, lo que demuestra que sí cumplen con los requerimientos técnicos de las plazas que se oferta en un $77.8 \%$.

Para el área de Gestión ambiental en un 75.4\%, en un 69.9 para el área de Prevención, control y remediación ambiental, en un 68.7\% para el área de Educación ambiental.

Todas las áreas deben ser reforzadas con competencias específicas para conseguir un mejor desempeño del egresado. 
Se sugiere un perfil reestructurado para los futuros egresados de la carrera de ingeniería ambiental con las siguientes características: Título: Ingeniero Ambiental con las áreas del perfil: Área de Recursos naturales, biodiversidad y de adaptación al cambio climático. Área de Sistemas de gestión ambiental. Área de Gestión de riesgos seguridad y salud ocupacional. Área de Administración, prevención, control y remediación ambiental. Área Formación y educación ambiental. Competencias genéricas y competencias específicas adecuadas e integrales.

\section{AGRADECIMENTOS}

A la Universidad Nacional «Santiago Antúnez de Mayolo». A los docentes de la Facultad de Ciencias del Ambiente, a los egresados de la Facultad de Ciencias del Ambiente, Escuela Profesional de Ingeniería Ambiental, a los empleadores.

\section{REFERENCIAS BIBLIOGRÁFICAS}

Bruner, Juan. 2010. «Aseguramiento de la calidad y nuevas demandas sobre la educación superior en América Latina». Revista Iberoamericana de Educación Superior. Vol.1, No 1. <http://www niversia.net/index.php/ries/article/viewArticle/2> [Consulta: 22-01-2014].

Comisión Central de Reestructuración Curricular. 2010. Guia del proceso de Reestructuración curricular de la Universidad «Santiago Antúnez de Mayolo». Documento de trabajo. Huaraz: Universidad Nacional «Santiago Antúnez de Mayolo».

Fuentes, Carlos. 2000. Modelo curricular con base a competencias profesionales. Santa Fe de Bogotá: Fundación Escuela Superior INPAHU.

Fundación Fórum Ambiental. 2001. Perfil profesional del responsable del medio ambiente en las organizaciones. Barcelona. <http://www.fundació/forumambiental.org.> [Consulta: 10-10-2013].

Hawes, Guti y Corvalan, Oscar. 2005. Construcción de un perfil profesional. Proyecto Mececesup Tal 0101. Documento de trabajo 1/2004. Talca: Universidad de Talca / Instituto de Investigación y Desarrollo Educacional.

Salas, Walter. 2005. «Formación por competencias en educación superior. Una aproximación conceptual a propósito del caso colombiano». Revista Iberoamericana de Educación. $\mathrm{N}^{\circ} 36 / 9$.

Sánchez, Katia. 2013. ¿Qué es perfil profesional? <http://espanol.answers.yahoo.com/ question/index?qid=20091021134419AAKjPcU.> [Consulta: 12-11-2011]. 
| Paula Falcón

Sota, Javier y otros. 2002. Diagnóstico de la Universidad Peruana: Razones para una nueva reforma universitaria. Lima: Comisión Nacional por la Segunda Reforma Universitaria.

Tobón, Sergio. 2006. Aspectos básicos de la formación basada en competencias. Talca: Proyecto Mesesup.

Fecha de recepción: 11 de noviembre 2015

Fecha de aceptación: 06 de diciembre 2015

\section{Correspondencia}

Paula Falcón Romero

paulafalc02@gmail.com 